Dept. of Math. Univ. of Oslo

Pure Mathematics No. 18

ISSN 0806-2439 ОстовеR 2006

\title{
SEMI-GODUNOV SCHEMES FOR GENERAL TRIANGULAR SYSTEMS OF CONSERVATION LAWS
}

\author{
K. H. KARLSEN, S. MISHRA, AND N. H. RISEBRO
}

\begin{abstract}
We consider general $m \times m$ triangular systems of conservation laws in one space dimension. These systems arise in applications like multiphase flows in porous media and are non-strictly hyperbolic. We device simple and efficient finite volume schemes of the Godunov type which are based on a local decoupling of the system into a series of single conservation laws with discontinuous coefficients and are hence termed Semi-Godunov schemes. These schemes are not based on the characteristic structure of the system. We derive some useful properties of the schemes and show several numerical experiments to demonstrate their robustness and computational efficiency.
\end{abstract}

\section{INTRODUCTION}

We are interested in the following $m \times m$ system of conservation laws in one space dimension:

$$
\left(u_{i}\right)_{t}+f_{i}\left(u_{1}, \ldots, u_{i}\right)_{x}=0, \quad i=1, \ldots, m .
$$

Let $U=\left(u_{1}, u_{2}, \ldots, u_{m}\right)$ denote the vector of unknowns and $f=\left(f_{1}, f_{2}, \ldots, f_{m}\right)$. Then the Cauchy problem for the above conservation law can be written as

$$
\left\{\begin{array}{l}
U_{t}+f(U)_{x}=0, \\
U(x, 0)=U_{0}(x) .
\end{array}\right.
$$

Note that (1.1) has a special structure with the evolution of the $i$-th variable $u_{i}$ being independent of the $m-i$ succeeding variables $\left(u_{i+1}, \ldots, u_{m}\right)$. The Jacobian matrix of $f$ is given by

$$
A=\partial f=\left(\begin{array}{cccc}
\frac{\partial f_{1}}{\partial u_{1}} & 0 & \ldots & 0 \\
\frac{\partial f_{2}}{\partial u_{1}} & \frac{\partial f_{2}}{\partial u_{2}} & \ldots & 0 \\
\ldots & \ldots . & \ddots & 0 \\
\frac{\partial f_{m}}{\partial u_{1}} & \frac{\partial f_{m}}{\partial u_{2}} & \ldots & \frac{\partial f_{m}}{\partial u_{m}}
\end{array}\right)
$$

The flux Jacobian is a lower triangular matrix and hence the system is termed a triangular system of conservation laws. The eigenvalues of the Jacobian (i.e., the wave speeds) are the diagonal entries of $A$ given by

$$
\lambda_{i}=\frac{\partial f_{i}}{\partial u_{i}}, \quad 1 \leq i \leq m .
$$

1991 Mathematics Subject Classification. 35L65, 65M06, 76S05.

Key words and phrases. Triangular systems, Flows in Porous Media, Godunov type schemes, Discontinuous flux.

KHK has been supported in part by an Outstanding Young Investigators Award from the Research Council of Norway. 
Therefore the system (1.1) is hyperbolic. Since the eigenvalues may not be distinct, the conservation law (1.2) is not strictly hyperbolic. The waves coincide for $U$ such that

$$
\lambda_{i}(U)=\frac{\partial f_{i}}{\partial u_{i}}(U)=\frac{\partial f_{j}}{\partial u_{j}}(U)=\lambda_{j}(U), \quad i \neq j .
$$

Conservation laws where the characteristic speeds may coincide are called resonant. This resonance may lead to a very complicated solution of the Riemann problem, thereby making it difficult to design numerical schemes based on solving Riemann problems. The main aim of this paper is to formulate simple and efficient numerical schemes of finite volume type for (1.1).

A special case for (1.1) is the $2 \times 2$ system:

$$
\begin{cases}\left(u_{1}\right)_{t}+f\left(u_{1}\right)_{x}=0, & (x, t) \in \mathbb{R} \times \mathbb{R}^{+}, \\ \left(u_{2}\right)_{t}+g\left(u_{1}, u_{2}\right)_{x}=0, & (x, t) \in \mathbb{R} \times \mathbb{R}^{+}, \\ \left(u_{1}, u_{2}\right)(x, 0)=\left(u_{1,0}(x), u_{2,0}(x)\right), & x \in \mathbb{R} .\end{cases}
$$

The above system has the same resonant structure as the more general system (1.1) and serves as a prototype for the general case. In Section 2, we argue that a conservation law of this type can serve as a simplified model of three phase flow in porous media.

As mentioned earlier, the solution of the Riemann problem for (1.1) is complicated on account of resonance. Most finite volume numerical schemes such as the Godunov or the Roe scheme are based on solving local (exact or approximate) Riemann problems are very difficult to formulate for (1.1). Hence, we need to consider numerical schemes that are not based on the characteristic structure of this system.

One possible choice is to use central schemes that do not rely on the characteristic structure. But, first order central schemes like the Lax-Friedrichs scheme or the Rusanov scheme are too dissipative to use in practice, although the situation can be remedied by using higher order central schemes. We will not be considering central schemes in this paper.

Another alternative is to devise upwind schemes which resolve the solutions satisfactorily even at first order. In this paper, we formulate a new class of such schemes that do not rely on the characteristic structure or solutions of the Riemann problems. Instead, we will locally (in time) solve only individual component equations of (1.1) as scalar conservation laws by treating the other variables as coefficients for the scalar equation. Hence, locally we reduce the system (1.1) to a set of scalar conservation laws with discontinuous coefficients on the form:

$$
\begin{cases}w_{t}+h(k(x, t), w)_{x}=0, & (x, t) \in \mathbb{R} \times \mathbb{R}^{+}, \\ w(x, 0)=w_{0}(x), & x \in \mathbb{R} .\end{cases}
$$

where $w$ is the conserved variable and the coefficient $k$ is allowed to be discontinuous along curves in the $(x, t)$ plane. The above approach can be easily illustrated for the prototype system (1.3). Indeed, one can see that since the evolution of $u_{1}$ is independent of $u_{2}$ in (1.3), we can evolve $u_{1}$ and treat it as a coefficient in the evolution equation for $u_{2}$, thus reducing (1.3) to an equation of the form (1.4). The same holds for the more general system (1.1). Equations of the form (1.4) have been studied widely in recent years and a fairly well developed theoretical and numerical treatment is available, see $[1,2,3,4,5,6,7,8,9]$ and the references cited therein for more details. 
This approach of deriving finite volume schemes for conservation laws by locally viewing each component equation as a scalar conservation law but with discontinuous coefficients was first explored in the case of the $2 \times 2$ system (1.3) in [10], and the resulting numerical schemes were termed semi-Godunov schemes. The purpose of this paper is to design semi-Godunov schemes for more general $m \times m$ triangular systems (1.1). The semi-Godunov schemes do not use Riemann solutions for the full system and are therefore independent of the characteristic structure of (1.1). In this way we avoid the obstacles posed by resonance. Furthermore, these schemes are very simple to implement.

We have organized the rest of this paper as follows, in section 2, we will describe a reduced three phase flow model where the system (1.3) arises. The semiGodunov schemes are defined in section 3 while numerical experiments involving these schemes are shown in section 4 and the contents of this paper are summarized in section 5

\section{A TRIANGULAR THREE-PHASE FLOW MODEL}

In this section we argue that triangular systems of conservation laws of the type (1.3) can be used as models for three phase flow in porous media.

Simulation of a variety of oil recovery processes involve models of three-phase flow in porous media. Often the three-phases of interest are oil, gas, and water. As a model we consider incompressible, immiscible three-phase flow in a one-dimensional homogeneous and isotropic reservoir (see, e.g., [11]). The oil, water, and gas saturations are given by $S_{o}, S_{w}, S_{g}$ respectively.

The mass conservation equation for phase $l=, w, o, g$ reads

$$
\phi\left(S_{l}\right)_{t}+\left(U_{l}\right)_{x}=0,
$$

where $\phi$ is the porosity of the medium and $U_{l}$ is the Darcy velocity or flow rate corresponding to each phase $l$. By Darcy's law, the flow rate is given by

$$
U_{l}=-k \lambda_{l}\left(\frac{\partial P_{l}}{\partial x}-G\right) \quad l=w, o, g,
$$

where $k$ denotes the absolute permeability of the medium, $\lambda_{l}$ is the mobility (relative permeability divided by viscosity) of phase $l, P_{l}$ is the pressure of phase $l$, and $G$ is the gravity term. We assume that the flow is incompressible i.e., the total flow

rate $q=\sum_{l=w, o, g} U_{l}$ is a constant. For the sake of simplicity, we assume that the differences in the capillary pressures between the phases are zero. This assumption is reasonable when the total flow rate is high (the flow is convection dominated).

By adding the mass conservation equations (2.1) and using the above assumptions, we arrive at the following $2 \times 2$ system of conservation laws:

$$
\left\{\begin{array}{l}
\left(S_{g}\right)_{t}+\left(F_{g}\left(S_{g}, S_{w}, S_{o}\right)\right)_{x}=0 \\
\left(S_{w}\right)_{t}+\left(F_{w}\left(S_{g}, S_{w}, S_{o}\right)\right)_{x}=0 \\
S_{g}+S_{w}+S_{o}=1
\end{array}\right.
$$

where the fluxes are given by,

$$
\begin{aligned}
& F_{g}\left(S_{g}, S_{w}, S_{o}\right)=\frac{q \lambda_{g}}{\lambda_{t}}+\frac{k}{\lambda_{t}} \lambda_{w} \lambda_{g}\left(g_{w}-g_{g}\right)+\frac{k}{\lambda_{t}} \lambda_{o} \lambda_{g}\left(g_{o}-g_{g}\right), \\
& F_{w}\left(S_{g}, S_{w}, S_{o}\right)=\frac{q \lambda_{w}}{\lambda_{t}}+\frac{k}{\lambda_{t}} \lambda_{w} \lambda_{g}\left(g_{g}-g_{w}\right)+\frac{k}{\lambda_{t}} \lambda_{o} \lambda_{w}\left(g_{o}-g_{w}\right),
\end{aligned}
$$


where $\lambda_{t}=\lambda_{o}+\lambda_{g}+\lambda_{w}$ is the total mobility.

It is well known that (2.2) can be a mixed type system, i.e., contain elliptic regions and thus fail to be hyperbolic. It is outside the scope of this paper to discuss this feature here. Instead we refer to [12] (and the references therein) for a review of some of the current views that exist today regarding mathematical and numerical theory for mixed type systems.

In many situations the mobility of the gas phase is much larger than that of the other phases. This means that the flux of gas is largely independent of whether the other phase is oil or water. As a consequence

$$
F_{g}\left(S_{g}, S_{w}, S_{o}\right)=\tilde{F}\left(S_{g}, 1-S_{g}\right)=\hat{F}\left(S_{g}\right) .
$$

Assuming this relationship, system (2.2) reduces to the following system

$$
\begin{aligned}
\left(S_{g}\right)_{t}+\left(\hat{F}_{g}\left(S_{g}\right)\right)_{x} & =0, \\
\left(S_{w}\right)_{t}+\left(F_{w}\left(S_{g}, S_{w}\right)\right)_{x} & =0 .
\end{aligned}
$$

The above equation is a special case of (1.1). We refer to [13] and the references cited therein for the model when capillary forces are included.

It is to be emphasized that that although the assumption of independence of the gas phase is not valid for all fractional flow functions, there exists a large class of fractional flow functions for which this assumption appears to be reasonable. In view of the fact that this assumption makes the model simpler and much more tractable, we can use this "reduced" model in several situations. A careful numerical study of this model (2.3) as an approximation to the full three-phase flow model needs to be carried out. An essential ingredient for this program is the development of efficient numerical schemes for (1.1).

We remark that a one dimensional model like the one that we are using is a good starting point for developing numerical schemes for the full three dimensional model where one can use the one dimensional numerical fluxes in directions normal to volume interfaces or along streamlines.

\section{Semi-Godunov schemes}

In this section, we describe the semi-Godunov finite volume schemes for the general $m \times m$ triangular system (1.1). We describe two schemes based on different approaches for solving single conservation laws with discontinuous coefficients.

3.1. Semi-Godunov (exact) scheme. We use a uniform space-time grid with space size $\Delta x$ and time step $\Delta t$. The discretization parameters are related by the standard CFL condition,

where

$$
\frac{\Delta t}{\Delta x} M \leq \frac{1}{2}
$$

$$
M=\max _{1 \leq i \leq m} \max _{a_{i} \in\left[s_{i}, S_{i}\right]} \frac{\partial f_{i}}{\partial u_{i}}\left(a_{1}, a_{2}, \ldots, a_{i}\right) .
$$

Let $t^{n}=n \Delta t$ and $x_{j}=j \Delta x$ for $n=0,1,2, \ldots$ and $j=\ldots,-1,-1 / 2,0,1 / 2,1, \ldots$. Let $I_{j}$ and $I^{n}$ denote the intervals

$$
I_{j}=\left[x_{j-1 / 2}, x_{j+1 / 2}\right), \quad I^{n}=\left[t^{n}, t^{n+1}\right) .
$$

Set

$$
\chi_{j}^{n}(x, t)=\chi_{I_{j}}(x) \chi_{I^{n}}(t),
$$


where $\chi_{\Omega}$ denotes the characteristic function of a set $\Omega$.

The initial data are discretized by taking cell averages:

$$
U_{j}^{0}=\frac{1}{\Delta x} \int_{x_{j-1 / 2}}^{x_{j+1 / 2}} U(x, 0) d x .
$$

Assuming that the approximate solution $U_{j}^{n}$ at the $n$-th time level are known, we have to determine the updates $U_{j}^{n+1}$ at the next time level. Next, we describe the algorithm for computing these updates.

The Algorithm.

Step 1. Consider the first component equation of (1.1) given by

$$
\left(u_{1}\right)_{t}+\left(f_{1}\left(u_{1}\right)\right)_{x}=0 .
$$

Since it is a scalar conservation law, we can update it by a standard Godunov-type flux to obtain the updates:

$$
u_{(1, j)}^{n+1}=u_{(1, j)}^{n}-\frac{\Delta t}{\Delta x}\left(F^{1}\left(u_{(1, j)}^{n}, u_{(1, j+1)}^{n}\right)-F^{1}\left(u_{(1, j-1)}^{n}, u_{(1, j)}^{n}\right)\right),
$$

where $F^{1}$ is the (scalar) Godunov flux with respect to the function $f_{1}$. For any function $h$, the Godunov flux $H$ is given by

$$
H(a, b)=\left\{\begin{array}{lc}
\min _{\theta \in[a, b]} h(\theta), & \text { if } a \leq b, \\
\max _{\theta \in[b, a]} h(\theta), & \text { otherwise. }
\end{array}\right.
$$

Note that the above flux is Lipschitz and is non-decreasing in the first variable and non-increasing in the second variable.

We define approximate solution $u_{i}^{\Delta x}$ on $\mathbb{R} \times \mathbb{R}^{+}$for $i=1, \ldots, m$ by setting

$$
u_{i}^{\Delta x}(x, t)=\sum_{n, j} \chi_{j}^{n}(x, t) u_{i, j}^{n}
$$

Step 2. Once we have the update for $u_{1}$, the next step is to consider the equation for the second component $u_{2}$, which reads

$$
\left(u_{2}\right)_{t}+\left(f_{2}\left(u_{1}, u_{2}\right)\right)_{x}=0 .
$$

Instead of viewing $u_{1}$ as an unknown in the above equation, we will treat it as a coefficient locally, i.e., we substitute $u_{1}^{\Delta x}\left(x, t^{n}\right)$ instead of $u_{1}$ in the above equation to obtain the approximate solution $u_{2}^{\Delta x}$ as a solutions of the local Riemann problem

$$
\begin{aligned}
& \left(u_{2}^{\Delta x}\right)_{t}+f_{2}\left(u_{(1, j)}^{n}, u_{2}^{\Delta x}\right)_{x}=0, \quad \text { if } x<x_{j+1 / 2}, \\
& \left(u_{2}^{\Delta x}\right)_{t}+f_{2}\left(u_{(1, j+1)}^{n}, u_{2}^{\Delta x}\right)_{x}=0, \quad \text { if } x>x_{j+1 / 2}, \\
& u_{2}^{\Delta x}\left(x, t^{n}\right)= \begin{cases}u_{(2, j)}^{n}, & x<x_{j+1 / 2}, \\
u_{(2, j+1)}^{n}, & x>x_{j+1 / 2} .\end{cases}
\end{aligned}
$$

Thus by locally freezing the unknown $u_{1}$ in the second equation of (1.1), we obtain a scalar conservation law with a discontinuous coefficient. We solve the Riemann problem (3.3) to obtain formulas for the interface fluxes.

We can solve the Riemann problem (3.3) exactly for a large class of fluxes. The details of the solution of the Riemann problem can be found in $[1,8]$. Using the 
Riemann solution to define interface fluxes, we obtain the updating formula

$$
\begin{aligned}
u_{(2, j)}^{n+1}=u_{(2, j)}^{n}-\frac{\Delta t}{\Delta x} & \left(F^{2}\left(u_{(1, j)}^{n}, u_{(1, j+1)}^{n}, u_{(2, j)}^{n}, u_{(2, j+1)}^{n}\right)\right. \\
& \left.-F^{2}\left(u_{(1, j-1)}^{n}, u_{(1, j)}^{n}, u_{(2, j-1)}^{n}, u_{(2, j)}^{n}\right)\right),
\end{aligned}
$$

with the numerical flux $F^{2}$ being defined from the solutions of the Riemann problem (3.3). Explicit formulas for $F^{2}$ can be given in a large number of cases (see below).

Step $i$ (for $i=3, \ldots, m$ ). The above strategy can be used at each step. At the $i$ th step, we substitute the variables $u_{k}^{\Delta x}, 1 \leq k \leq i-1$, in place of the unknowns $u_{k}, 1 \leq k \leq i-1$, in the evolution equation for $u_{i}$ to obtain the following Riemann problem at the interface $x_{j+1 / 2}$ for $j \in \mathbb{Z}$ :

$$
\begin{aligned}
& \left(u_{i}^{\Delta x}\right)_{t}+f_{i}\left(u_{(1, j)}^{n}, \ldots, u_{(i-1, j)}^{n}, u_{i}^{\Delta x}\right)_{x}=0, \quad \text { if } x<x_{j+1 / 2}, \\
& \left(u_{i}^{\Delta x}\right)_{t}+f_{i}\left(u_{(1, j+1)}^{n}, \ldots, u_{(i-1, j)}^{n}, u_{i}^{\Delta x}\right)_{x}=0, \quad \text { if } x>x_{j+1 / 2}, \\
& u_{i}^{\Delta x}\left(x, t^{n}\right)= \begin{cases}u_{(i, j)}^{n}, & x<x_{j+1 / 2}, \\
u_{(i, j+1)}^{n}, & x>x_{j+1 / 2} .\end{cases}
\end{aligned}
$$

We can solve the above Riemann problem exactly to obtain the updating formula

$$
\begin{aligned}
u_{(i, j)}^{n+1}=u_{(i, j)}^{n}-\frac{\Delta t}{\Delta x}[ & F^{i}\left(u_{(1, j)}^{n}, u_{(1, j+1)}^{n}, \ldots, u_{(i-1, j)}^{n}, u_{(i-1, j+1)}^{n}, u_{(i, j)}^{n}, u_{(i, j+1)}^{n}\right) \\
& \left.-F^{i}\left(u_{(1, j-1)}^{n}, u_{(1, j)}^{n}, \ldots, u_{(i-1, j-1)}^{n}, u_{(i-1, j)}^{n}, u_{(i, j-1)}^{n}, u_{(i, j)}^{n}\right)\right] .
\end{aligned}
$$

The numerical fluxes $F^{i}$ are defined from the solutions of the Riemann problem. We can provide explicit formulas for the numerical fluxes in several cases. For example, if the flux $f_{i}$ satisfies the hypothesis

$$
\begin{aligned}
& a \mapsto f_{i}\left(a_{1}, a_{2}, \ldots, a_{(i-1)}, a\right) \text { has at most one minimum (resp. maximum) } \\
& \text { and no maxima (resp. minima) in } \mathbb{R} \text { for all } a_{j} \in \mathbb{R} \text { with } j=1, \ldots, i .
\end{aligned}
$$

In this case we have

$$
\begin{aligned}
F^{i}\left(a_{1}, b_{1}, \ldots, a_{i-1}, b_{i-1}, a_{i}, b_{i}\right) \\
\quad=\max \left\{f_{i}\left(a_{1}, \ldots, a_{i-1}, \max \left(\theta_{a}, a_{i}\right)\right), f_{i}\left(b_{1}, \ldots, b_{i-1}, \min \left(\theta_{b}, b_{i}\right)\right)\right\} .
\end{aligned}
$$

By repeating the procedure for all the $m$ components of $U$, we obtain the so-called semi-Godunov (exact) scheme, which we will refer to as the $S G(E)$ scheme. The updating formula for this scheme can be written compactly as

$$
U_{j}^{n+1}=U_{j}^{n}-\frac{\Delta t}{\Delta x}\left(F\left(U_{j}^{n}, U_{j+1}^{n}\right)-F\left(U_{j-1}^{n}, U_{j}^{n}\right)\right),
$$

with the $U_{j}^{n}=\left(u_{(1, j)}^{n}, \ldots, u_{(i, j)}^{n}, \ldots, u_{(m, j)}\right)$ being the vector of unknowns and $F=$ $\left(F^{1}, \ldots, F^{i}, \ldots, F^{m}\right)$ the numerical flux vector. 
3.2. Semi-Godunov (Approximate) scheme. Our second scheme is similar to the semi-Godunov (Exact) scheme, except instead of solving Riemann problems for scalar conservation laws with discontinuous coefficients exactly, we will provide approximate solutions of them.

We use the same grid and CFL condition as for the $S G(E)$ scheme. The initial data are discretized by taking cell averages:

$$
V_{j}^{0}=\frac{1}{\Delta x} \int_{x_{j-1 / 2}}^{x_{j+1 / 2}} U(x, 0) d x .
$$

Let the approximate solution $V_{j}^{n}$ at the $n$-th time level be known. The goal is to determine the approximate solution $V_{j}^{n+1}$ at the succeding time level. We describe next the algorithm for computing $V_{j}^{n+1}$.

The Algorithm.

Step 1. The first step is similar to Step 1 in the derivation of the $S G(E)$ scheme. The update $v_{(1, j)}^{n+1}$ is given by

$$
v_{(1, j)}^{n+1}=v_{(1, j)}^{n}-\frac{\Delta t}{\Delta x}\left(\bar{F}^{1}\left(v_{(1, j)}^{n}, v_{(1, j+1)}^{n}\right)-\bar{F}^{1}\left(v_{(1, j-1)}^{n}, v_{(1, j)}^{n}\right)\right),
$$

where $\bar{F}^{1}$ is the Godunov flux with respect to the function $f_{1}$. We define the approximate solution $v_{i}^{\Delta x}$ on $\mathbb{R} \times \mathbb{R}^{+}$for $i=1, \ldots, m$ by setting

$$
v_{i}^{\Delta x}(x, t)=\sum_{n, j} \chi_{j}^{n}(x, t) v_{i, j}^{n}
$$

Step 2. Similarly to Step 2 in the derivation of the $S G(E)$ scheme, we substitute the approximate solution $v_{1}^{\Delta x}$ instead of $u_{1}$ in the evolution equation for $u_{2}$ to get the Riemann problem

$$
\begin{aligned}
& \left(v_{2}^{\Delta x}\right)_{t}+f_{2}\left(v_{(1, j)}^{n}, v_{2}^{\Delta x}\right)_{x}=0, \quad \text { if } x<x_{j+1 / 2}, \\
& \left(v_{2}^{\Delta x}\right)_{t}+f_{2}\left(v_{(1, j+1)}^{n}, v_{2}^{\Delta x}\right)_{x}=0, \quad \text { if } x>x_{j+1 / 2}, \\
& v_{2}^{\Delta x}\left(x, t^{n}\right)= \begin{cases}v_{(2, j)}^{n}, & x<x_{j+1 / 2}, \\
v_{(2, j+1)}^{n}, & x>x_{j+1 / 2} .\end{cases}
\end{aligned}
$$

Rather than solving the above Riemann problem exactly, we can update $v_{2}^{\Delta x}$ by using an approximate Riemann solution. The simplest way to build an approximate solver is to use the exact solution of the following Riemann problem:

$$
v_{2}^{\Delta x}+f_{2}\left(\frac{\left(v_{(1, j)}^{n}+v_{(1, j+1)}\right)}{2}, v_{2}^{\Delta x}\right)_{x}=0, \quad v_{2}^{\Delta x}\left(x, t^{n}\right)= \begin{cases}v_{(2, j)}^{n}, & x<x_{j+1 / 2}, \\ v_{(2, j+1)}^{n}, & x>x_{j+1 / 2} .\end{cases}
$$

This is a Riemann problem for a single conservation law without discontinuities in the coefficient. This strategy leads to the staggered schemes of $[3,4,5]$. The arithmetic average is simplest choice for an equivalent flux, although other choices can be considered. Fluxes resulting from the above Riemann solution yield the following updating formula:

$$
\begin{aligned}
v_{(2, j)}^{n+1}=v_{(2, j)}^{n}-\frac{\Delta t}{\Delta x} & {\left[\bar{F}^{2}\left(v_{(1, j)}^{n}, v_{(1, j+1)}^{n}, v_{(2, j)}^{n}, v_{(2, j+1)}^{n}\right)\right.} \\
& \left.-\bar{F}^{2}\left(v_{(1, j-1)}^{n}, v_{(1, j)}^{n}, v_{(2, j-1)}^{n}, v_{(2, j)}^{n}\right)\right] .
\end{aligned}
$$


where the interface flux $\bar{F}^{2}\left(a_{1}, b_{1}, a_{2}, b_{2}\right)$ corresponds to the standard Godunov flux with respect to the function $f_{2}\left(\frac{a_{1}+b_{1}}{2}, \cdot\right)$.

Step $i(i=3, \ldots, m)$. The above strategy can be repeated for every step. At the $i$-th step, we can use the approximate solution to the Riemann problem (3.5) by arithmetic averaging of the adjacent fluxes to obtain the following updating formula:

$$
\begin{aligned}
v_{(i, j)}^{n+1}=v_{(i, j)}^{n}-\frac{\Delta t}{\Delta x} & {\left[\bar{F}^{i}\left(v_{(1, j)}^{n}, v_{(1, j+1)}^{n}, \ldots, v_{(i-1, j)}^{n}, v_{(i-1, j+1)}^{n}, v_{(i, j)}^{n}, u_{(i, j+1)}^{n}\right)\right.} \\
& \left.-\bar{F}^{i}\left(v_{(1, j-1)}^{n}, v_{(1, j)}^{n}, \ldots, v_{(i-1, j-1)}^{n}, v_{(i-1, j)}^{n}, v_{(i, j-1)}^{n}, v_{(i, j)}^{n}\right)\right],
\end{aligned}
$$

where the interface flux $\bar{F}\left(a_{1}, b_{1}, \ldots, a_{i-1}, b_{i-1}, a_{i}, b_{i}\right)$ is the Godunov flux corresponding to the flux function $f_{i}\left(\frac{a_{1}+b_{1}}{2}, \ldots, \frac{a_{i-1}+b_{i-1}}{2}, \cdot\right)$.

By combining the fluxes derived from the approximate solutions of the Riemann problems for the corresponding conservation laws with discontinuous fluxes (that is, updates like (3.10)), we obtain the so-called semi-Godunov (approximate) scheme, which we will refer to as the $S G(A)$ scheme. The updating formula for this scheme reads

$$
V_{j}^{n+1}=V_{j}^{n}-\frac{\Delta t}{\Delta x}\left(\bar{F}\left(V_{j}^{n}, V_{j+1}^{n}\right)-\bar{F}\left(V_{j-1}^{n}, V_{j}^{n}\right)\right),
$$

where $V_{j}^{n}=\left(v_{(1, j)}^{n}, \ldots, v_{(i, j)}^{n}, \ldots, v_{(m, j)}\right)$ and $\bar{F}=\left(\bar{F}_{1}, \ldots, \bar{F}_{i}, \ldots, \bar{F}_{m}\right)$.

We remark that one can show that both of these schemes have several desirable properties. It is straightforward to show that the numerical flux functions are consistent, i.e.,

$$
F(V, V)=f(V) .
$$

Furthermore, in many cases one has $L^{\infty}$ bounds on the approximations generated by these semi-Godunov schemes. In particular this holds if there are numbers $s_{i}<S_{i}, i=1, \ldots, m$, such that

$$
f_{i}\left(a_{1}, \ldots, a_{i-1}, s_{i}\right)=\text { const. and } f_{i}\left(a_{1}, \ldots, a_{i-1}, S_{i}\right)=\text { const. },
$$

for all $a_{1}, \ldots, a_{i-1}$. In this case we have that

$$
U_{j}^{0} \in \Pi_{i=1}^{m}\left[s_{i}, S_{i}\right] \text { for all } j \Longrightarrow U_{j}^{n} \in \Pi_{i=1}^{m}\left[s_{i}, S_{i}\right] \text { for all } j .
$$

Another case where the approximations remain bounded I have made the changes suggested by Nils Henrik. THeis if

$$
\left|f_{i}\left(a_{1}, \ldots, a_{i-1}, a\right)\right| \geq C \log (|a|+1),
$$

for all $i$ and all sufficiently large $|a|$, and where the constant does not depend on $a_{1}, \ldots, a_{i-1}$. In this case there are constants $M$ and $N$ such that

$$
\left|u_{i, j}^{n}\right| \leq M e^{N n \Delta t}, \text { for all } n>0 \text { and all } i \text { and } j \text {. }
$$

The proof of statements (3.12) and (3.13) involve a detailed study of the solution of the Riemann problem for conservation laws with discontinuous flux, see [9]. 


\section{NUMERICAL EXPERIMENTS}

As mentioned earlier, both the $S G(E)$ and $S G(A)$ schemes are very easy to implement since explicit formulas are available for the numerical fluxes. The computational cost is similar to solving $m$ uncoupled scalar equations and is comparable to that of the Lax-Friedrichs scheme. However, these schemes are less dissipative than the Lax-Friedrichs scheme. In this section, we present a few numerical experiments to illustrate the robustness of these schemes.

Example 1. We use the flux functions

$$
f_{1}\left(u_{1}\right)=\frac{1}{2} u_{1}^{2}, \quad f_{2}\left(u_{1}, u_{2}\right)=4 u_{1} u_{2}\left(u_{2}-1\right),
$$

and the Riemann initial data

$$
u_{1}(x, 0)=\left\{\begin{array}{ll}
0.75, & x<0, \\
0.25, & x \geq 0,
\end{array} \quad u_{2}(x, 0)=0.5 .\right.
$$

In this simple model problem, we know the exact solution:

$$
u_{1, \mathrm{ex}}(x, t)=\left\{\begin{array}{ll}
3 / 4 & x<t / 2, \\
1 / 4 & x \geq t / 2,
\end{array} \quad u_{2, \mathrm{ex}}(x, t)= \begin{cases}1 / 2 & x<-t, \\
5 / 6 & -t \leq x<t / 2, \\
1 / 2 & x \geq t / 2 .\end{cases}\right.
$$

In Figure 1 we show the computed solutions for $u_{2}$ at $t=0.75$ using the $S G(E)$ and the $S G(A)$ schemes with $\Delta x=1 / 20$ in the interval $[-1,1]$. From this it seems that the both schemes perform equally well, and this impression is confirmed by other computations. As expected, the schemes have some numerical diffusion at the shocks, yet the shocks speeds are captured quite accurately. Since we have a

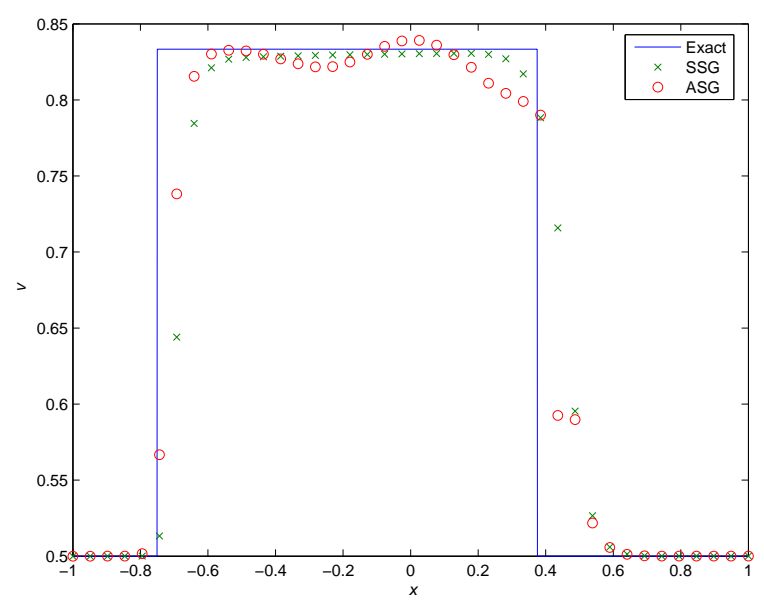

FIGURE 1. Example 1, the SG(E)- and the SG(A)-scheme approximating $u_{2}$ with $\Delta x=1 / 20$ and $t=0.75$.

formula for the exact solution in this case, we have computed the relative errors in 
the $L^{1}$ norm for various $\Delta x$. The relative errors are defined as

$$
e=\frac{\sum_{j}\left|u_{2}^{\Delta x}\left(x_{j}, t\right)-u_{2, \mathrm{ex}}^{\Delta x}\left(x_{j}, t\right)\right|}{\sum_{j}\left|u_{2, \mathrm{ex}}^{\Delta x}\left(x_{j}, t\right)\right|}
$$

where $u_{2, \text { ex }}$ is the exact solution (stated above) and $t=0.75$. These errors are reported in Table 1. From this table it seems that both schemes are first order

\begin{tabular}{c|ccccccc}
$n$ & 3 & 4 & 5 & 6 & 7 & 8 & 9 \\
\hline SG(A) & 8.6 & 5.1 & 2.8 & 1.4 & 0.7 & 0.4 & 0.018 \\
SG(E) & 7.6 & 3.7 & 2.1 & 1.1 & 0.6 & 0.3 & 0.015
\end{tabular}

TABLE 1. $100 \times$ Relative $L^{1}$ error for the SSG and ASG schemes.

We used $\Delta x=2^{-n}$ in the interval $[-1,1]$.

convergent. We have tested both the schemes on several such model problems and obtained similar results.

Example 2. To test the applicability of the triangular model as a model of threephase flow in porous media, we have compared the results obtained by the triangular and the full model on a water flooding problem. This also serves as a good test case for the efficiency of the schemes designed in this paper. We use the relative permeabilities

$$
\lambda_{g, w, o}=\frac{1}{\nu_{g, w, o}} S_{g, w, o}^{2}
$$

with $S_{i}$ denoting the saturation of phase $i$, and $\nu_{i}$ the viscosity. We have used the following viscosities

$$
\nu_{g}=1, \quad \nu_{w}=80 \quad \text { and } \quad \nu_{o}=100 .
$$

In addition we have set

$$
\rho_{g}=1 / 20, \quad \rho_{w}=1 \quad \text { and } \quad \rho_{o}=9 / 10,
$$

and have set the gravitational constant and the absolute permeability to unity. This gives the flux functions

$$
\begin{aligned}
& F_{g}(u, v)=\frac{u^{2}}{u^{2}+v^{2} / 100+(1-v-u)^{2} / 80}\left(1-\frac{17 v^{2}}{200}-\frac{19(1-v-u)^{2}}{160}\right), \\
& F_{o}(u, v)=\frac{v^{2}}{100 u^{2}+v^{2}+(5 / 4)(1-v-u)^{2}}\left(1+\frac{v^{2}}{10}+\frac{19(1-v-u)^{2}}{20}\right),
\end{aligned}
$$

where we have set $u=S_{g}$ and $v=S_{o}$. This is the "full" three phase flow model, and we see that $F_{g}$ is not very dependent on $v$. In order to define a triangular method we set

which gives the flux function

$$
v=\frac{1-u}{2} \quad \text { and } \quad(1-u-v)=\frac{1-u}{2}
$$

$$
F_{g}(u)=\frac{u^{2}}{u^{2}+(9 / 1600)\left(1-u^{2}\right)}\left(1-\frac{163}{3200}(1-u)^{2}\right) .
$$

We have used the initial values

$$
v(x, 0)=\left\{\begin{array}{ll}
0, & x<0, \\
\frac{1}{2}+\frac{1}{4} \sin (2 \pi x), & x \geq 0,
\end{array} \quad u(x, 0)= \begin{cases}0, & x<0 \\
1-v(x, 0), & x>0\end{cases}\right.
$$


This is meant to model the situation where one has a mixture of oil and gas in the reservoir, and one attempts to inject water in order to force out the oil and the gas. We have used (4.2) for the gas-flux and the SG(E) scheme to calculate approximate solutions. In Figure 2 we show contour plots of the gas, oil, and water saturations as functions of $x$ and $t$ for $-0.05 \leq x \leq 5$ and $0 \leq t \leq 2$. Again the solutions are captured quite well.
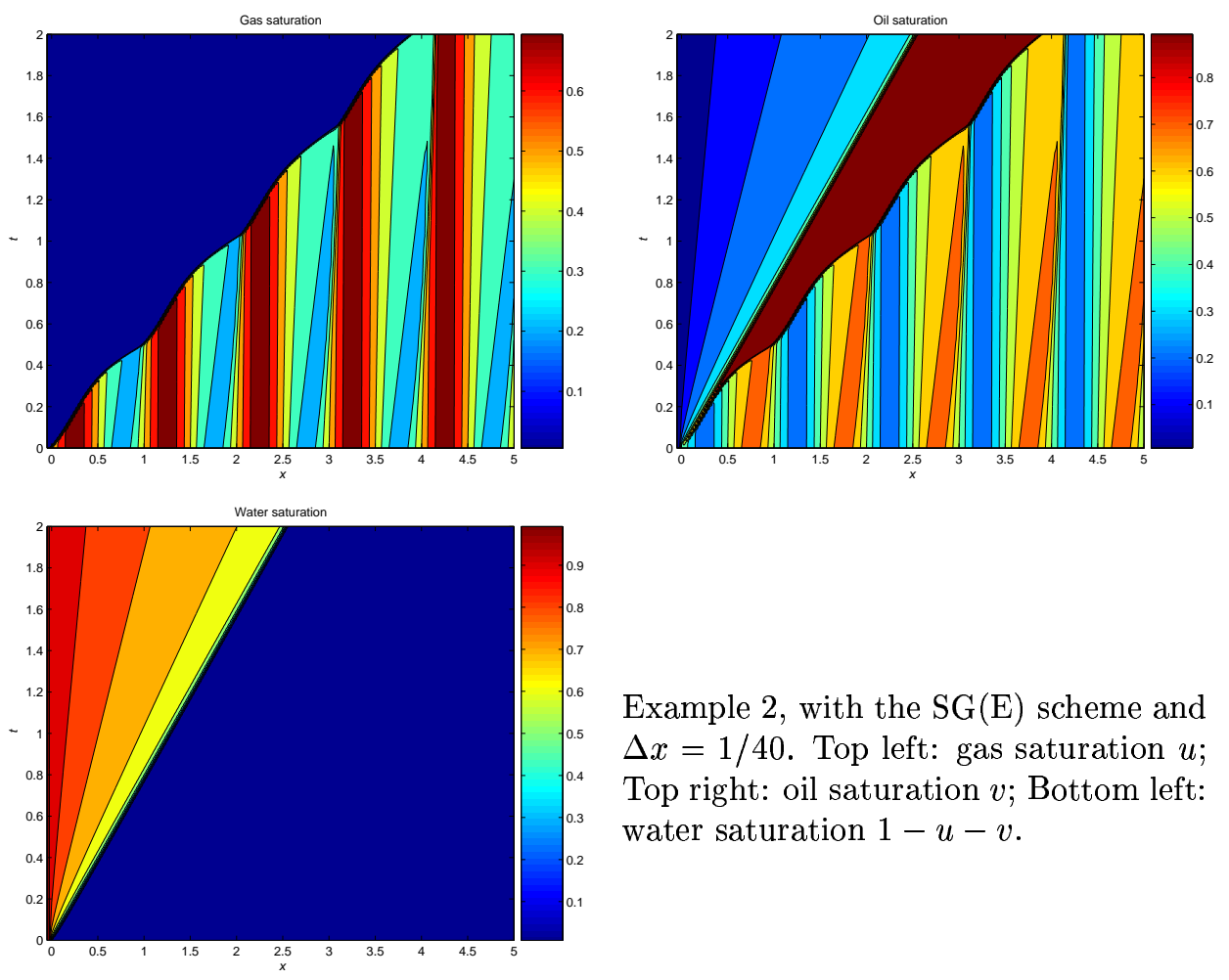

Example 2, with the SG(E) scheme and $\Delta x=1 / 40$. Top left: gas saturation $u$; Top right: oil saturation $v$; Bottom left: water saturation $1-u-v$.

FIGURE 2

Example 3. So far we have considered numerical examples for $2 \times 2$ triangular systems. Next, we present some numerical results for a model $3 \times 3$ triangular system, based on the flux functions

$$
\begin{aligned}
f_{1}\left(u_{1}\right) & =\frac{1}{2} u_{1}^{2}, \\
f_{2}\left(u_{1}, u_{2}\right) & =u_{1} u_{2}\left(u_{2}-2\right), \\
f_{3}\left(u_{1}, u_{2}, u_{3}\right) & =u_{1} u_{2}\left(u_{3}-1\right)\left(u_{3}-2\right),
\end{aligned}
$$

and the Riemann initial data

$$
\begin{aligned}
& u_{1}(x, 0)=\left\{\begin{array}{ll}
2, & \text { if } \quad x<0, \\
0.5, & \text { if } \quad x>0,
\end{array} \quad u_{2}(x, 0)=\left\{\begin{array}{lll}
1.8, & \text { if } \quad x<0, \\
1.2, & \text { if } \quad x>0,
\end{array}\right.\right. \\
& u_{3}(x, 0)=\left\{\begin{array}{lll}
1.9, & \text { if } \quad x<0, \\
1.1, & \text { if } \quad x>0 .
\end{array}\right.
\end{aligned}
$$


The numerical results for the $\mathrm{SG}(\mathrm{E})$ and $\mathrm{SG}(\mathrm{A})$ schemes are shown in Figure 3. As expected, both the schemes do quite well in resolving the solution. In particular,
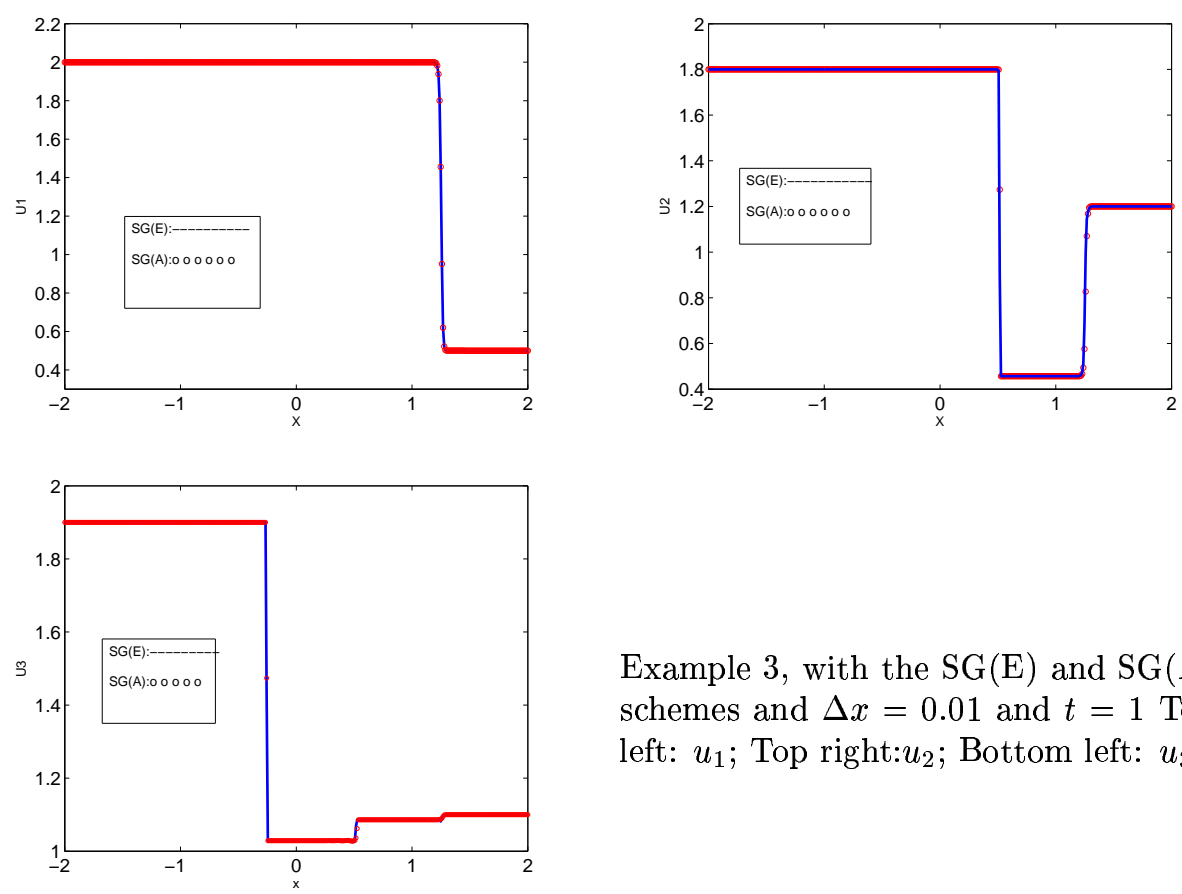

Example 3, with the SG(E) and SG(A) schemes and $\Delta x=0.01$ and $t=1$ Top left: $u_{1}$; Top right: $u_{2}$; Bottom left: $u_{3}$.

\section{FIGURE 3}

the resolution of $u_{3}$ is quite good despite the fact that the leading shock on the right is very weak.

Example 4. We use the same fluxes as in the previous example (i.e., (4.4)), but we replace the initial data by

$$
u_{1}(x, 0)= \begin{cases}2, \text { if } x<0, & u_{2}(x, 0) \equiv 1.5, \\ 0.5, \quad \text { if } x>0, & u_{3}(x, 0) \equiv \frac{11}{6}\end{cases}
$$

This problem is more complicated than the previous example as the initial data is chosen to induce resonance. All the three eigenvalues coincide in this case. We show the results in Figure 4. The key point in this example is the complicated structure of $u_{3}$ in the form of a compound shock (shock followed by rarefaction) in the second wave for $u_{3}$. This feature is a consequence of the resonance of the initial data in this case and was absent in the previous example. Notice the resolution of the compound shock by both schemes in this case. There is a slight undershoot in the rightmost shock wave for the SG(E) scheme, but the undershoot vanishes in the limit. We have also tested the Lax-Friedrichs scheme for this example and found that we need twenty to thirty times more mesh points to get the same resolution as with the SG(E) and SG(A) schemes. 

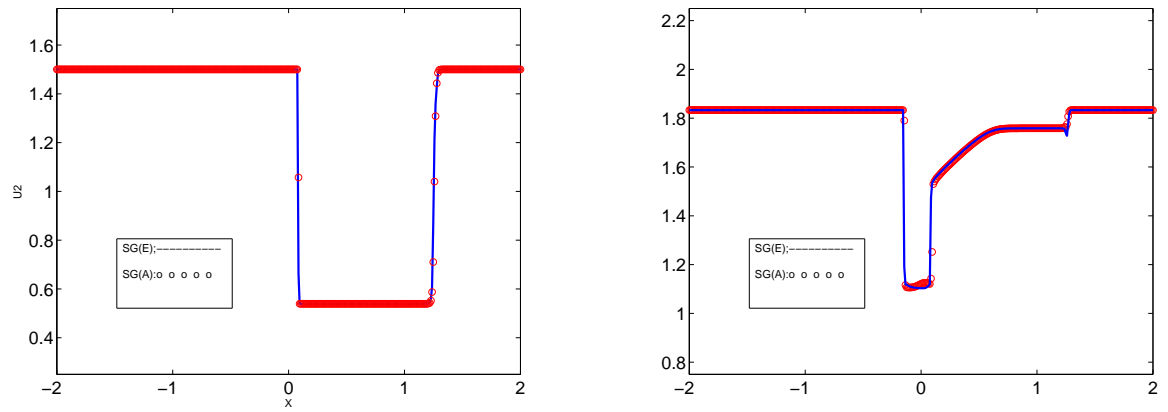

Figure 4. Example 4, with the $\mathrm{SG}(\mathrm{E})$ and $\mathrm{SG}(\mathrm{A})$ schemes, $\Delta x=$ 0.01 , and $t=1$. Left: $u_{2}$; Right: $u_{3}$.

\section{Conclusion}

The main issue addressed in this paper has been the design of numerical schemes for general $m \times m$ triangular systems of conservation laws. One motivation for considering equations in triangular form is due to their appearance as special "reduced" models for three-phase flows in a porous medium. Although, these systems are hyperbolic, they fail to be strictly hyperbolic because the eigenvalues can coincide. Hence, they posses a complicated wave structure making the design of conventional numerical schemes quite hard. We have described a class of finite-volume schemes, termed semi-Godunov schemes, which are based on "local" decoupling of the system into a sequence of single conservation laws with discontinuous coefficients. Two classes of schemes of this type have been presented. One class is based on exact Riemann solvers for conservation laws with discontinuous flux (cf. the SG(E) scheme (3.7)), while another class is based on approximate Riemann solvers for conservation laws with discontinuous flux (cf. the SG(A) scheme (3.11)).

Both schemes are easy to implement. The computational cost for the $m \times m$ system is the same as solving $m$ scalar conservation laws simultaneously and is similar to the cost of the Lax-Friedrichs schemes. Yet, these schemes have low numerical diffusion and resolve the solution quite well. We can prove some properties of the schemes, including consistency, invariant region principles, and moreover that they reduce to the standard upwind schemes when the flux functions are monotone. Hence, these schemes can be considered as extensions of the upwind scheme for systems with non-monotone fluxes. We have presented numerical experiments to justify the robustness and computational efficiency of the schemes.

Since the schemes rely on solvers for scalar conservation laws (with discontinuous flux), it is easy to design higher order versions. Similarly, schemes of this type can be easily modified to incorporate effects of heterogeneous media. Finally, these schemes can form the basis for computing numerical fluxes along normal directions in a multi-dimensional finite volume code.

\section{REFERENCES}

[1] T.Gimse and N.H.Risebro. Solution of Cauchy problem for a conservation law with discontinuous flux function. SIAM J. Math. Anal, 23 (3): 635-648, 1992. 
[2] S. Diehl. A conservation law with point source and discontinuous flux function modeling continuous sedimentation. SIAM J. Appl. Math., 56 (2):1980-2007, 1995.

[3] J.D.Towers. Convergence of a difference scheme for conservation laws with a discontinuous flux. SIAM J. Numer. Anal.,38(2):681-698, 2000.

[4] J.D. Towers. A difference scheme for conservation laws with a discontinuous flux-the nonconvex case. SIAM J. Numer. Anal.,39(4): 1197-1218, 2001.

[5] K. H. Karlsen, N.H.Risebro and J.D.Towers. Upwind difference approximations for degenerate parabolic convection-diffusion equations with a discontinuous coefficient. IMA J. Numer. Anal., 22(4):623-664, 2003.

[6] K. H. Karlsen, N.H.Risebro and J.D.Towers. $L^{1}$ stability for entropy solution of nonlinear degenerate parabolic convection-diffusion equations with discontinuous coefficients. Skr. K. Nor. Vidensk. Selsk. ,3, 2003, 49 pages.

[7] Adimurthi, J. Jaffré, and G. D. Veerappa Gowda. Godunov-type methods for conservation laws with a flux function discontinuous in space. SIAM J. Numer. Anal., 42(1):179-208, 2004.

[8] Adimurthi, Siddhartha Mishra and G.D.Veerappa Gowda. Optimal entropy solutions for conservation laws with discontinuous flux. Journal of Hyp. Diff. Eqns., 2(4): 1-56, 2005.

[9] S. Mishra. Analysis and Numerical approximation of conservation laws with discontinuous coefficients $P h D$ Thesis, Indian Institute of Science, Bangalore, 2005.

[10] K.H. Karlsen, S. Mishra and N.H. Risebro. Convergence of finite volume schemes for a triangular system of conservation laws. Preprint, 2006.

[11] G. Chavent and J.Jaffre. Mathematical models and Finite elements for Reservoir simulation. North Holland, Amsterdam, 1986.

[12] R. Bürger, K. H. Karlsen, E. M. Tory, and W. L. Wendland. Model equations and instability regions for the sedimentation of polydisperse suspensions of spheres. ZAMM Z. Angew. Math. Mech., 82(10):699-722, 2002.

[13] K. H. Karlsen, K.-A. Lie, J. R. Natvig, H. F. Nordhaug, and H. K. Dahle. Operator splitting methods for systems of convection-diffusion equations: nonlinear error mechanisms and correction strategies. J. Comput. Phys., 173(2):636-663, 2001.

(Kenneth Hvistendahl Karlsen)

Centre of Mathematics for Applications (CMA)

UNIVERSITY OF OSLO

P.O. Box 1053, BLINDERN

N-0316 OSLO, NoRWAY

E-mail address: kennethk@math.uio.no

$U R L:$ http://www.math.uio.no/ ${ }^{\sim}$ kennethk/

(Siddhartha Mishra)

Centre of Mathematics for Applications (CMA)

UNIVERSITY OF OSLO

P.O. Box 1053, BLINDERN

N-0316 Oslo, Norway

E-mail address: siddharm@cma.uio.no

(Nils Henrik Risebro)

Centre of Mathematics for Applications (CMA)

UNIVERSITY OF OSLO

P.O. BOX 1053, BLINDERN

N-0316 OSLo, Norway

E-mail address: nilshr@math.uio.no 